\title{
The Contribution of Speech-Evoked Cortical Auditory Evoked Potentials to the Diagnosis and Measurement of Intervention Outcomes in Children with Auditory Processing Disorder
}

\author{
Mridula Sharma, Ph.D., ${ }^{1}$ Suzanne C. Purdy, Ph.D., ${ }^{2}$ and \\ Andrea S. Kelly, Ph.D.2,3
}

Cortical auditory evoked potentials (CAEPs) are included in guidelines for assessment of auditory processing disorder (APD), but their diagnostic value and their use as a measure of treatment effectiveness have not been fully explored. The current study has three main aims: (1) to assess if there are differences in CAEPs recorded in quiet and in noise in children with APD compared to a control group of typically developing (TD) children, (2) to investigate the test-retest reliability of CAEPs, and (3) to determine whether participation in discrimination or language with or without support of personal frequency modulation (FM) resulted in significant changes in auditory function as measured by CAEPs. Fifty-five children with APD and 22 TD children age 7 to 13 years participated. There were group differences between TD children and children with APD for CAEP P1 and N250 amplitudes. CAEPs assessed on two occasions (separated by 7 to 10 days) showed a significant reduction in N250 amplitude on the second visit, highlighting the importance of establishing a stable baseline for CAEPs to more clearly delineate the effects of training from other factors influencing measurements. Participants with APD were randomly assigned to five treatment groups, including a no training group, two groups receiving discrimination training (one of these also used personal FM), and two groups receiving language training (one of these also used personal FM). N250 amplitudes changed during the baseline

${ }^{1}$ Audiology Section, Macquarie University, Sydney, Australia; ${ }^{2}$ Speech Science, The University of Auckland; ${ }^{3}$ Department of Audiology, Auckland District Health Board, Auckland, New Zealand.

Address for correspondence: Mridula Sharma, Ph.D., Department of Linguistics, Room C5A 518, Macquarie University, NSW 2109, Australia

(e-mail: mridula.sharma@mq.edu.au).
New Evidence in Auditory Processing Research; Guest Editor, Deborah Moncrieff, Ph.D.

Semin Hear 2014;35:51-64. Copyright (C) 2014 by Thieme Medical Publishers, Inc., 333 Seventh Avenue, New York, NY 10001, USA. Tel: +1(212) 584-4662. DOI: http://dx.doi.org/10.1055/s-0033-1363524.

ISSN 0734-0451. 
period, in the absence of any specific training, and hence a control group alone is insufficient for establishing intervention effectiveness; establishing a stable pretraining baseline for electrophysiological (and other) outcome measurements is also important. After 6 weeks training, children with APD in the discrimination treatment group showed a further significant change in N250 amplitudes, beyond the change that occurred during the baseline period. These results are limited by the small numbers of participants in each treatment group; however, the finding of some CAEP changes after training is consistent with published evidence for cortical changes after short-term auditory training.

KEYWORDS: Cortical auditory evoked potentials, noise, auditory processing disorder, test-retest reliability, auditory training, personal frequency modulation, signal-to-noise ratio

Learning Outcomes: As a result of this activity, the participant will be able to describe the features of cortical auditory evoked potentials in school-aged children and list the differences in cortical auditory evoked potentials between typically developing children and children with auditory processing disorder.

\section{CORTICAL AUDITORY EVOKED POTENTIALS AS A DIAGNOSTIC TOOL IN CHILDREN WITH POOR AUDITORY PROCESSING}

Cortical auditory evoked potentials (CAEPs) are responses from the cortex that can be elicited by speech tokens in infants, children, and adults. ${ }^{1-3}$ Auditory stimulus presentation generally results in $\mathrm{P} 1-\mathrm{N} 250$ peaks in children (6 to 12 years), ${ }^{3-5}$ although adultlike P1-N1-P1 peaks are evident when very slow presentation rates are used. ${ }^{6,7}$ CAEPs reflect the functional integrity of the auditory pathways and are regarded as a neurophysiological correlate of speech processing ability. ${ }^{8}$ Evidence for this comes from studies showing an association between speech perception ability and CAEP characteristics and from data indicating disrupted CAEPs in people with lesions involving central auditory pathways., ${ }^{9,10}$

CAEP latencies and/or amplitudes are affected in children with learning disability or language delay, ${ }^{3,11,12}$ especially when stimuli are presented in continuous background noise. ${ }^{4,13,14}$ Children with auditory perceptual deficits have difficulty perceiving speech in noise, ${ }^{15}$ thus it is anticipated that they may show a greater impact of noise on CAEPs than typically developing (TD) children, as demonstrated by Cunningham et al..$^{5}$ Although guidelines for diagnosis of auditory processing disorder (APD) recommend the inclusion of objective electrophysiological measures for some assessments, ${ }^{16}$ there is still a lack of standardization of CAEP recording parameters (e.g., stimulus type and duration, type and level of background noise), and there is a paucity of normative CAEP data in TD children. Also, only a few studies have measured CAEPs in children with a clinical diagnosis of APD. ${ }^{12}$ The current study contributes to this literature by comparing CAEPs in children diagnosed with APD to results for TD children for a speech sound in quiet and in noise.

\section{ARE CAEPs SENSITIVE TO AUDITORY TRAINING EFFECTS?}

CAEPs have been used in training studies to assess neural plasticity in children and adults and hence it is possible that CAEPs may be sensitive to treatment effects in adults and children with APD. Researchers have examined longer-term auditory training effects such as the effects of musical training, short-term passive listening effects such as occurs during repeated 
auditory testing, and short-term active auditory training effects in experiments where participants respond to the auditory stimuli. N1 amplitude increases after only 1 hour of participation in a frequency discrimination task. ${ }^{17} \mathrm{~N} 1$ and $\mathrm{P} 2$ amplitudes are larger in adult musicians than in age-matched nonmusicians, ${ }^{18}$ and this is presumed to reflect long-term auditory training effects. $\mathrm{N} 1$ and $\mathrm{P} 2$ amplitudes also are enhanced significantly by short-term auditory training in adults. ${ }^{19}$ Most studies report enhanced CAEP amplitudes; however, Reinke et al found that a week of training on novel vowels was associated with enhanced P2 amplitude and shortened N1 and P2 latencies in adults age 19 to 34 years. ${ }^{20}$ Consistent with training studies in adults, there is some evidence that CAEP amplitudes and latencies can be altered by auditory training in children with auditory processing disorders or learning difficulties. ${ }^{4,13,14}$

Use of personal frequency modulation (FM) systems may have an auditory training effect through greater exposure to everyday acoustic signals. For example, positive effects of FM use on CAEP amplitudes in noise have been reported. ${ }^{4}$ Personal FM systems also could have an auditory training effect as a result of enhanced quality of the acoustic signal. In one study of children with auditory processing difficulties, P2 amplitude was significantly bigger in the experimental group of children who wore FM systems for a year compared with an age-matched control group who did not wear FM systems. ${ }^{21}$ The primary aim of the current study was to evaluate whether CAEPs are sensitive to training effects in children with APD. CAEPs may be neither necessary nor important to measure training effects; however, they do provide information about the underlying neurophysiology. ${ }^{22}$ Objectivity is the primary advantage of CAEPs over behavioral measures of training effectiveness.

Before training effects can be evaluated, test-retest reliability needs to be established for CAEPs. Stable test-retest results have been reported for CAEPs recorded in adults ${ }^{1}$; however, there are few if any such studies in children. Hence another aim of the current study was to evaluate the test-retest stability of CAEP peaks in quiet and in noise prior to auditory training in children with APD. The overall aims of the current research were to investigate: (1) CAEPs in quiet versus noise (+3-dB signal-to-noise ratio) in children with APD and compare these results to those for TD children, (2) CAEP test-retest variations in children with APD, and (3) the effect of auditory training with and without use of FM devices on CAEPs in children with APD.

\section{METHODS}

\section{Participants}

Participants with suspected APD were recruited from a range of sources including audiologists in public and private settings, special educators, speech pathologists, tutors, educational psychologists, teachers, and parents. Ninety potential participants with suspected APD were assessed using tests of auditory processing, cognition, sustained attention, auditory memory, receptive and expressive language, reading, phonological awareness, and CAEPs. All participants with suspected APD had hearing thresholds better than 20-dB hearing loss (HL) and normal type A tympanograms. These assessments occurred over one to three sessions, each lasting 3 hours, separated by approximately 1 week (no more than 10 days). ${ }^{2,24}$ Twentytwo TD children (control) with no listening or learning concerns (mean age 10.7 years, standard deviation [SD] 1.7, range 7 to $12 ; 12$ girls, 10 boys) also participated in the electrophysiological testing on one occasion only. TD control children had hearing thresholds better than 20dB HL, normal tympanometry, present acoustic reflexes, and passed the Dichotic Digits and Frequency Pattern auditory processing tests.

\section{APD Diagnosis}

Participants were given five behavioral tests that assess a range of auditory processes: Dichotic Digit Test (DDT) Version 2, Frequency Pattern Test (FPT), Random Gap Detection Test, compressed (45\%) and reverberated (0.3 seconds) hearing in noise test (HINT) sentences, and the $500-\mathrm{Hz}$ tone masking level difference. These assessments are described in more detail by Sharma et al. $^{23}$ Participants were diagnosed 
with APD when their performance was two SD below the mean on one or more APD tests. Fiftyfive children who met this diagnostic criterion for APD (36 boys, 19 girls) age 7 to 13 years (mean 9.7 years, SD 1.4, range 9 to 12 years) participated in the electrophysiology and training study. Characteristics of the 55 participants with APD are described previously. ${ }^{24}$ Performance of the children with APD was poorest overall on the FPT and DDT tasks. The majority of participants with APD (62\%) had additional reading and language difficulties whereas $4 \%$ of participants had a diagnosis of APD only. The remainder had either language difficulties or reading deficits, but not both, in addition to APD.

\section{Electrophysiological Assessments}

Electrophysiological measurements were performed using a Neuroscan Inc. SCAN (version 4.2) (Compumedics Limited, Victoria, Australia) evoked potential system. Neuroscan Inc. STIM 2 software was used to generate white noise and to present the speech phoneme $/ d a /$ in quiet at $70-\mathrm{dB}$ sound pressure level and in noise at $+3-\mathrm{dB}$ signal-to-noise ratio. Electrophysiological testing was performed in a soundproof booth. The participant was seated in a comfortable reclining chair, watching a self-chosen movie with subtitles on and the sound muted. Participants were instructed to sit still and relax. The tester was seated outside the soundproof booth and monitored the participant's state via a window between the soundproof booth and the control room. The overall duration of each electroencephalogram (EEG) session including electrode placement and electrophysiological testing was 40 minutes.

Cortical responses were elicited using a 158-millisecond-long speech phoneme / da/ recorded from an Australian female speaker (/a/ as in the word hard; the low back $a$ is spoken without the $r$ sound in the word hard in Australian English). Stimuli were presented via STIM $10 \mathrm{ohm}$ (Compumedics, Victoria, Australia) EART insert earphones and link foam tips in the participants' ears bilaterally, with an interstimulus interval of 910 milliseconds. Data were collected in two blocks of 200 to 250 EEG trials per stimulus condition, with noise and quiet blocks randomized. CAEPs were recorded from frontal $(\mathrm{Fz})$ and vertex $(\mathrm{Cz})$ midline electrodes with left and right mastoids as reference electrodes and ground on the high forehead. Electrode impedances were less than $5 \mathrm{k} \Omega$. EEG channels were amplified (gain 500) and filtered (0.1 to $100 \mathrm{~Hz}$ ) online.

\section{Training}

Children in the APD group $(n=55)$ were pseudorandomly distributed into five groups receiving no training (no training group, $n=12$ ) or training (four groups, $n=43$ ) receiving either discrimination or language training, with or without personal FM systems. Details of the training were reported by Sharma et al. ${ }^{24}$ The training was undertaken in a research laboratory 1 hour per week for 6 weeks and each week participants received worksheets that needed to be done for 15 minutes a day. Personal FM systems were provided to the children in two of the groups to wear at school. Discrimination training had a bottom-up focus and encompassed basic discrimination tasks such as tonal frequency, temporal, or intensity discrimination. Language-based training had a top-down focus and included metacognitive strategies. ${ }^{24}$

\section{Data Analysis}

Offline analysis involved converting continuous EEG data to single-trial event related potential (ERP) waveforms with a time window of -100 to 600 milliseconds (with 100 milliseconds prestimulus baseline correction). ERPs were then digitally filtered $(30 \mathrm{~Hz}$ low pass, 24 $\mathrm{dB}$ /octave). Prior to averaging, ERPs contaminated by eyeblinks producing voltage variations exceeding $\pm 100 \mathrm{~V}$ were rejected. There were $\sim 300$ to 450 EEG trials per average for each stimulus condition for each participant. P1 and N250 peak latencies and amplitudes were visually identified for the average waveforms for each condition. If peaks were present across electrode positions, then amplitude, latency, and morphology was expected to be approximately consistent across $\mathrm{Fz}$ and $\mathrm{Cz}$ electrode positions. This was used as a guide only when peak picking as some variations in amplitudes and latencies might occur between frontal/ central electrodes. ${ }^{25} \mathrm{P} 1$ was identified as 
occurring within the range 40 to 70 milliseconds. N250 was identified as the maximum negativity between 100 and 300 milliseconds.

Analyses of variance (ANOVAs) were performed to determine effects of noise on latency and amplitude data for the P1 and N250 peaks, with age as a covariate and electrode location $(\mathrm{Fz}, \mathrm{Cz})$ as a repeated measure. Main effects and interactions were regarded as significant if $p<0.05$. Planned comparisons were performed only when significant main effects or interactions were obtained. Separate ANOVAs were undertaken to determine: (1) whether TD control and APD group CAEPs differed in quiet and noise, (2) whether CAEPs showed test-retest changes during a 7 - to $10-$ day baseline pretraining period in children with APD, and (3) whether 6 weeks of discrimination or language training with or without personal FM use was associated with changes in CAEPs in children with APD.

\section{RESULTS}

The three components of the study addressed three main questions:

1. Are there differences in /da/-CAEPs between children diagnosed with APD and the TD control group with no listening or learning concerns and do differences occur in both quiet and in noise?

2. Are there test-retest effects on CAEPs in children with APD that could reduce the usefulness of CAEPs as a measure of auditory training effectiveness?

3. Is the use of personal FM systems and discrimination or language training over a 6-week period associated with significant changes in CAEP latencies and amplitudes?

\section{Are There Differences in /da/-CAEPs between Children Diagnosed with APD and TD Control Group and Do Differences Occur in Both Quiet and in Noise?}

Fig. 1 shows grand average CAEP waveforms for the APD and TD control groups in quiet and in noise. Statistical analyses included $\mathrm{Fz}$ and $\mathrm{Cz}_{\mathrm{z}}$ data; as no electrode differences were evident, data are presented for $\mathrm{Cz}$. There was a significant effect of noise on N250 latency and amplitudes; noise reduced N250 amplitude for both groups $(\mathrm{F}[1,74]=9.7, p=0.003)$. N250 latency was longer in noise for both groups $(\mathrm{F}[1,74]=20.1, p<0.0001)$. There were no significant group differences for P1 and N250 peak latencies or N250 amplitudes, but there was a significant overall group difference for P1 amplitude ( $\mathrm{F}[1,74]=6.3$, $p=0.014)$. N250 amplitude showed a significant interaction between group and stimulus condition $(\mathrm{F}[1,74]=9.9, p=0.002)$. Planned comparisons showed no group differences in N250 amplitudes for the noise condition, but there was a significant group difference in quiet $(p=0.035)$. This finding was unanticipated, as one would expect the greatest differences between TD control participants and those with APD to be in noise. Fig. 1 shows that there were CAEP differences between TD and APD waveforms in both quiet and noise. The standard errors listed in Table 1 indicate substantial N250 latency variation in noise for both groups, hence the lack of statistical difference for the noise condition may reflect greater intersubject variability in waveform morphology in noise.

\section{Are There Test-Retest Effects on CAEPs in Children with APD That Could Reduce the Usefulness of CAEPs as a Measure of Auditory Training Effectiveness?}

CAEP test and retest visits were within 7 to 10 days of each other for the 55 participants with APD. Repeated-measures ANOVA showed no latency differences between visits for $\mathrm{P} 1$ or N250. Significant test-retest differences were seen for N250 amplitudes, however. There was a significant interaction between stimulus condition and visit for $\mathrm{N} 250$ amplitude ( $\mathrm{F}[1,52]$ $=11.5, \quad p=0.001)$. Planned comparisons showed that N250 amplitudes were significantly smaller at visit 2 compared to visit 1 for both quiet $(p<0.0001)$ and noise $(p=0.014)$ conditions; however, as shown in Figs. 2 and 3, testretest differences were much greater in quiet than in noise. For both visits 1 and 2 , there was a significant overall effect of noise on N250 amplitudes $(\mathrm{F}[1,48]=5.6, p=0.023)$. 

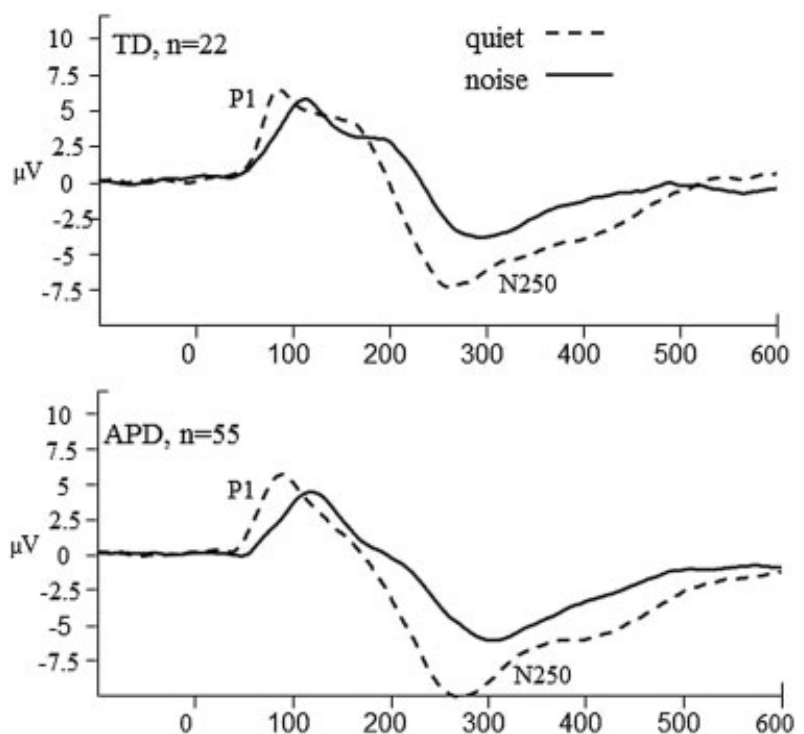

Figure 1 /da/ in quiet and in noise (3-dB signal-to-noise ratio) in TD and APD group at Fz (right ear reference). APD, auditory processing disorder; TD, typically developing.

Is the Use of Personal FM Systems and Discrimination or Language Training over a 6-Week Period Associated with Significant Changes in CAEP Latencies and Amplitudes?

The 55 children in the APD group underwent electrophysiological assessment on three occasions: twice during the baseline period to assess test-retest effects on CAEPs as described above, and once after a 6-week training block. Participants with APD were randomly assigned to five groups for the training phase of the study. A no training group of 12 children with APD received no intervention but were assessed in the same way as the other APD groups. The other four groups received different types of training: discrimination $(n=12)$, discrimina- tion plus $\mathrm{FM}(n=10)$, language $(n=12)$, language plus $\mathrm{FM}(n=9)$; as described in Sharma et al. ${ }^{24}$ N250 amplitudes changed significantly overall across the three visits (two baseline visits and one posttraining; $\mathrm{F}[1,100]=3.5, p=0.027)$. To evaluate whether the APD no training group versus combined intervention groups showed different patterns of change across the three visits, planned comparisons were undertaken. These showed a significant difference between visits 2 and 3 in quiet $(p=0.008)$ but not in noise and between baseline visits 1 and 2 both in quiet and in noise $(p<0.001)$ for the combined intervention groups (Fig. 4). There were differences between visit 1 and 2 for each of the APD intervention groups. The APD no training

Table 1 Average P1 and N250 Latencies and Amplitudes for the TD Control Group Children and the Children with APD Tested at Visit 1, for the Cz-Right Mastoid Electrode Montage

\begin{tabular}{|c|c|c|c|c|c|}
\hline \multirow[t]{2}{*}{ Stimulus Condition } & \multirow[t]{2}{*}{ Group } & \multicolumn{2}{|c|}{ P1 } & \multicolumn{2}{|c|}{ N250 } \\
\hline & & Latency & Amplitude & Latency & Amplitude \\
\hline \multirow[t]{2}{*}{ Quiet } & TD control $(n=22)$ & $86.82(4.14)$ & $5.59(0.72)$ & $280.45(3.55)$ & $-10.16(0.74)$ \\
\hline & $\operatorname{APD}(n=55)$ & $90.55(2.71)$ & $4.85(0.43)$ & $284.24(7.23)$ & $-7.73(0.53)$ \\
\hline \multirow[t]{2}{*}{ Noise } & TD control $(n=22)$ & 110.09 (4.32) & $6.03(0.69)$ & $288.00(13.25)$ & $-5.37(0.45)$ \\
\hline & $\operatorname{APD}(n=55)$ & $109.62(3.35)$ & $5.14(0.42)$ & $306.69(5.92)$ & $-6.00(0.39)$ \\
\hline
\end{tabular}

None of the analyses showed a significant electrode effect. Numbers in parentheses are standard errors. APD, auditory processing disorder; TD, typically developing. 


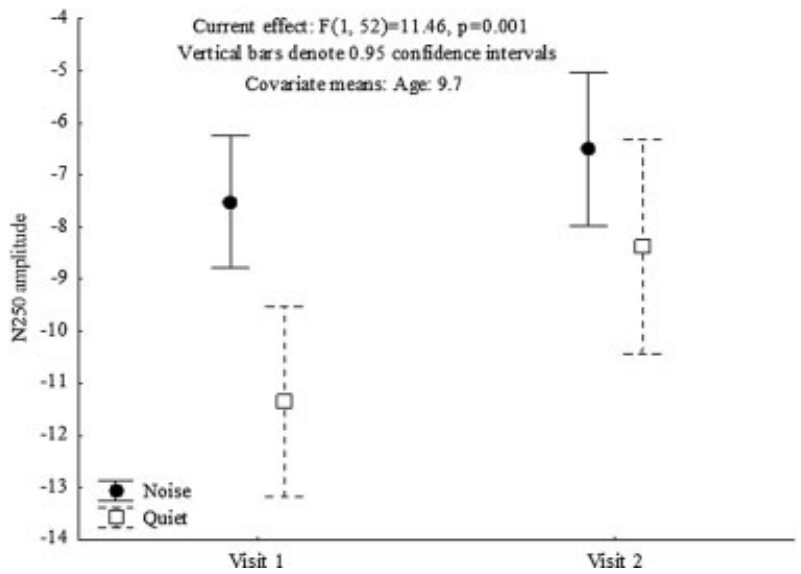

Figure 2 Significant difference was seen for N250 amplitude between two baselines for the APD group $(n=55)$. APD, auditory processing disorder.

group did not show significant differences between visits 2 and 3, but were similar to the intervention groups in showing a reduction in N250 amplitude between baseline visits 1 and 2 in quiet $(p=0.044)$, but not in noise. Thus, test-retest effects on N250 amplitudes during the baseline period were evident for the APD no training and intervention groups, but only the intervention groups showed a further change after training. In general, statistically significant test-retest changes and changes after the training period were more consistently seen in quiet than in noise. This could reflect greater intersubject variability of CAEP morphology in noise, as evidenced by greater N250 latency variance in noise (Table 1). The lack of a stable
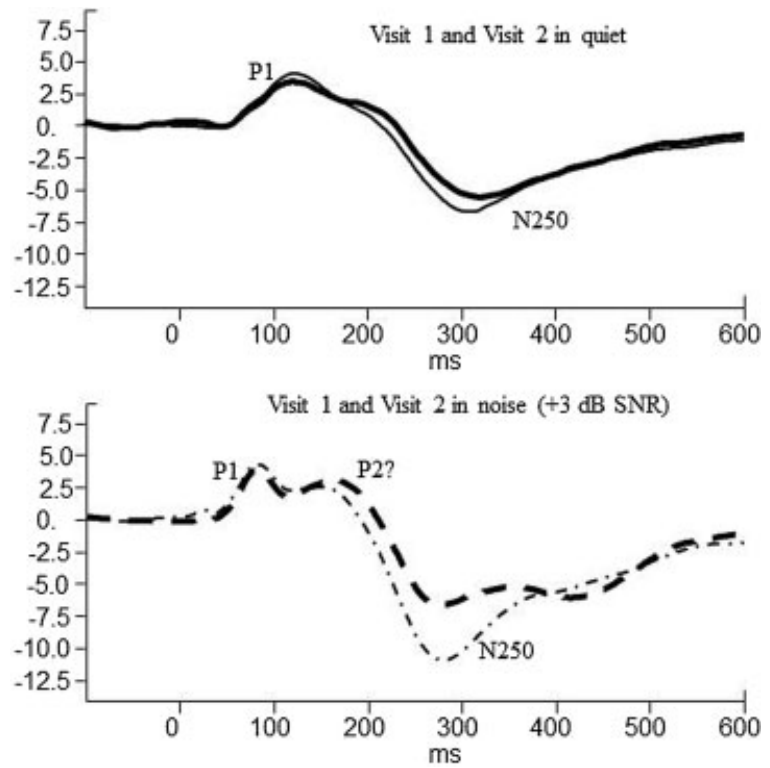

Figure 3 CAEPs responses for the APD group $(n=55)$ across the two visits, occurring within 10 days of each other, for both quiet and noise condition (Fz, right reference). Significant difference was seen for N250 amplitude between two baselines for the APD group. Visit 1 (thin lines) showed significantly bigger N250 in noise (dashed line) when compared with visit 2 (thick lines). APD, auditory processing disorder; CAEPs, cortical auditory evoked potentials. 


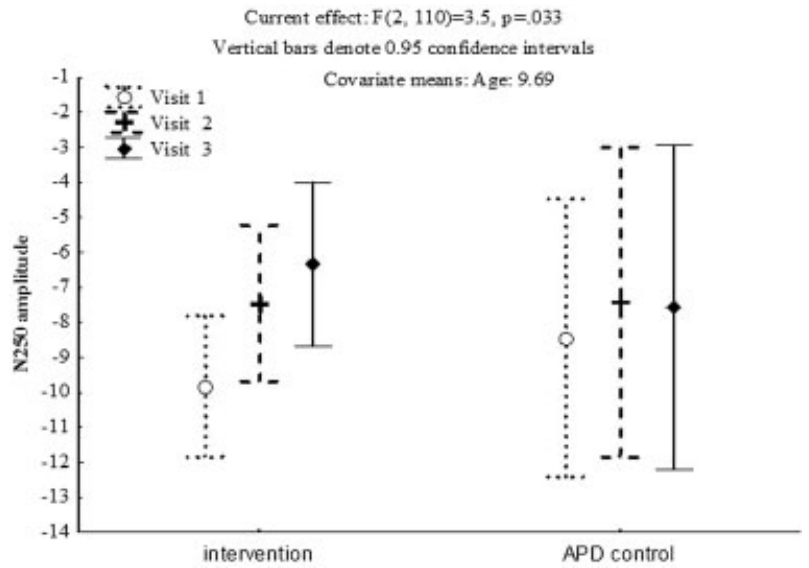

Figure 4 Comparing the N250 amplitude across three visits for the control $(n=12)$ and the combined intervention group (discrimination, language, discrimination and language with FM, $n=43$ ). Planned comparisons showed that the groups were significantly different between visit 1 and visit 2 (small and long dash lines, respectively) but only the intervention group was significantly different between visit 2 and 3 (long dash and continuous line, respectively). APD, auditory processing disorder.

CAEP baseline makes interpretation of possible training effects problematic; however, testretest changes are also of interest as they suggest that passive auditory training effects may occur in children with APD just through repeated exposure to the test stimuli. We would expect these test-retest differences to also occur in TD children, but these test-retest differences were not investigated for the TD control group. This is an area for future research.

Planned comparisons were undertaken to determine if different types of intervention had differing effects on CAEPs. After the training period, visit 2 versus 3 differences across both quiet and noise conditions were only significant for the discrimination group $(p=0.041)$ and showed a statistical trend for discrimination plus FM group $(p=0.073)$. There was also a visit 2 versus 3 statistical trend for the language group $(p=0.074)$. These results are limited by the small sample sizes for the separate intervention groups but do suggest a greater trend for N250 change across the intervention period for the two groups receiving discrimination training than for the two groups receiving language training (see Table 2 and Fig. 5).

\section{DISCUSSION}

\section{Effects of Noise on CAEPs in Children with APD and TD Controls}

As has been reported previously, ${ }^{26-28}$ noise resulted in longer N250 latencies and smaller N250 amplitudes, irrespective of the group. P1 was not affected by the noise. Irrespective of

Table 2 Average N250 Amplitudes for the 55 Children in the APD Group, Divided into Groups According to Training Type

\begin{tabular}{|c|c|c|c|c|c|c|}
\hline \multirow[t]{2}{*}{ Visit } & \multicolumn{2}{|c|}{ APD Control $(n=12)$} & \multicolumn{2}{|c|}{$\begin{array}{c}\text { Discrimination Training } \\
\text { with/without FM }(n=21)\end{array}$} & \multicolumn{2}{|c|}{$\begin{array}{c}\text { Language Training with/ } \\
\text { without FM }(n=21)\end{array}$} \\
\hline & Quiet & Noise & Quiet & Noise & Quiet & Noise \\
\hline & $-11.34(0.88)$ & $-6.11(0.69)$ & $-12.46(1.02)$ & $-8.70(0.62)$ & $-7.40(0.40)$ & $-11.71(0.86)$ \\
\hline & $-8.05(0.56)$ & $-7.80(1.07)$ & $-9.54(0.85)$ & $-6.96(0.58)$ & $-8.42(0.89)$ & $-6.48(0.70)$ \\
\hline & $-8.03(1.18)$ & $-5.90(1.14)$ & $-5.54(0.54)$ & $-7.44(0.94)$ & $-7.42(0.69)$ & $-6.48(0.59)$ \\
\hline
\end{tabular}

Results are shown for the two baseline visits ( 1 and 2 ) and the postintervention visit 3 and are for the $\mathrm{Cz}$-right mastoid electrode montage (none of the analyses showed a significant electrode effect). Numbers in parentheses are standard errors. APD, auditory processing disorder. 


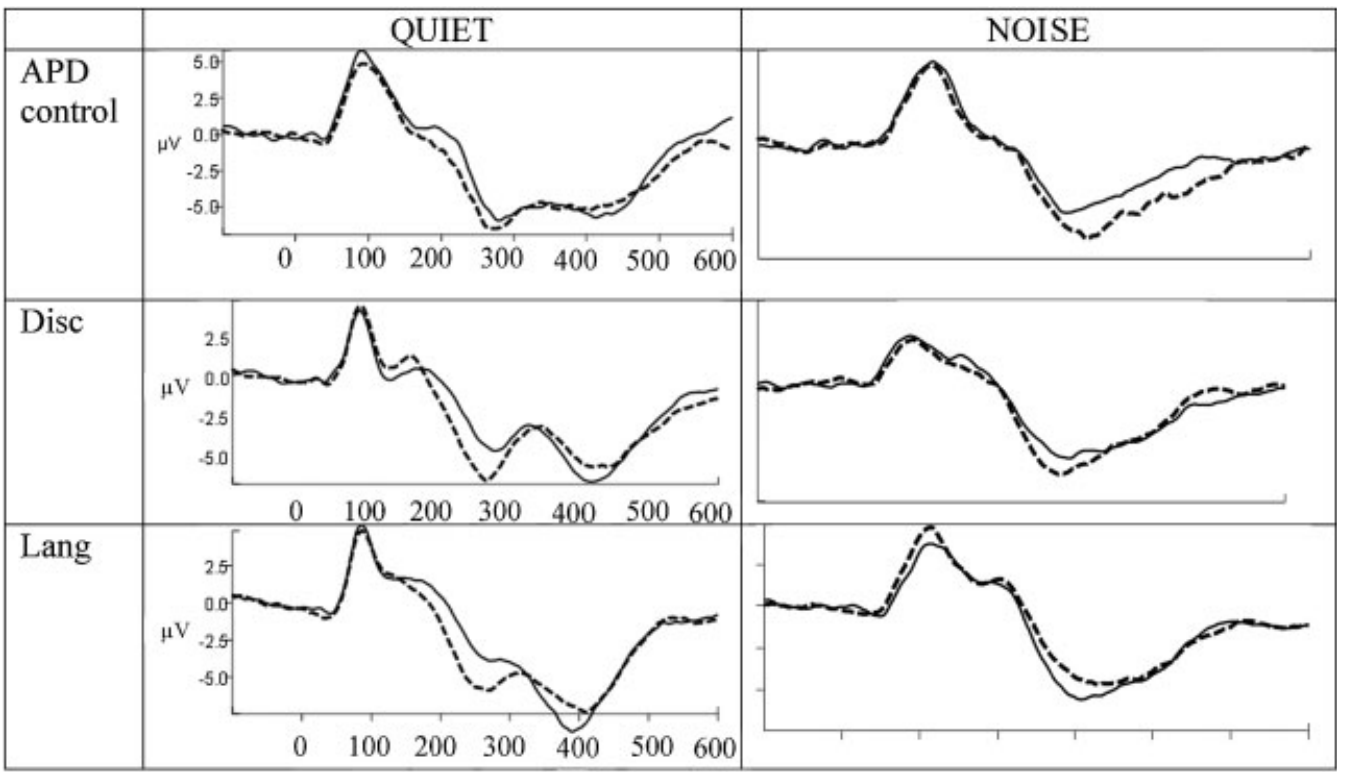

Figure 5 CAEPs comparing visit 2 (dashed line) and visit 3 (solid line) for three of the intervention groups in quiet and in noise at $\mathrm{Fz}$, right reference. The waveforms are similar for visit 2 and 3 in noise but discrimination (disc) training shows N250 amplitude to be significantly smaller whereas language (lang) training showed a trend for the quiet condition. APD, auditory processing disorder; CAEPs, cortical auditory evoked potentials.

group, N250 latency was longer in the presence of noise. This has been reported previously and may be a consequence of masking effects reducing speech sound audibility and neural synchrony. ${ }^{26,29}$ In the current study, N250, but not P1, was affected by noise. Previous studies in adults also have reported differential effects of noise on P1, N1, and P2 latencies and amplitudes. $^{27}$ Different CAEP components may exhibit differential effects of training, if, for example, training effects are mediated by attention. There are well-established effects of attention on $\mathrm{N} 1$ and $\mathrm{P} 2$ amplitudes, ${ }^{30,31}$ and auditory training effects have been reported for $\mathrm{N} 1$, N1-P2, or P2. ${ }^{4,32,33}$

The differential effect of noise on N250 in the current study highlights differences in the auditory processing and sources reflected in different CAEP components. P1 is believed to reflect the sensory representation of an acoustic stimulus at the cortical level. ${ }^{34,35}$ As long as the signal is perceived at the cortical level, P1 should be present. This is consistent with Billings et al's finding that tone-evoked P1 latencies, but not amplitudes, were affected by varying signal-to-noise ratio. ${ }^{27}$
The appearance of the N250 component in CAEPs recorded in children reflects complex effects of factors such as interstimulus interval and maturation. ${ }^{36}$ Ponton et al proposed that changes in the late negativity in the CAEPs of school-aged children reflect maturational changes in the more superficial cortical layers in the auditory cortex. Thus, the susceptibility of N250 to noise disruption seen in the current study may reflect the relative immaturity of N250 generators compared with P1. Ponton et al noted that it is possible that the generators of $\mathrm{P} 1$ are "adult-like by age 10 or perhaps even much younger". ${ }^{37(p .218)}$ This difference in the maturational time course of $\mathrm{P} 1$ and N250 may account in part for $\mathrm{P} 1$ being relatively resistant to the effects of noise.

\section{Differences in CAEPs between Children with APD and TD Controls}

P1 amplitude was smaller in children with APD compared to the TD control group. Purdy et al ${ }^{12}$ found shorter tone-evoked P1 latencies in children with APD, but no group effects on P1 amplitude. Cunningham et al found that all 
CAEP components were smaller for their learning problem group compared to normal hearing controls. ${ }^{14}$ There have been differences in CAEP results due to the use of speech versus nonspeech stimuli and also depending on whether participation selection criteria specifically excluded children with comorbid diagnoses such as autism. ${ }^{38-43}$ Another study that examined children with language-based learning problems showed that at least one subset of children had reduced CAEP amplitudes, especially for P1. ${ }^{3}$ It may be that reduced P1 amplitude is indicative of poorer sound representation at the level of the auditory cortex and that this is part of the auditory processing deficit. ${ }^{39}$ A reduction in $\mathrm{P} 1$ amplitude in children with auditory processing difficulties and other related difficulties is consistent with recent studies highlighting changes in cortical thickness in children with autism ${ }^{44-46}$ and the reported association between brain structure (cortical thickness, white matter microstructure, connectivity) and evoked potential and behavioral measures of auditory processing. ${ }^{46-48}$

\section{Group Differences in Quiet versus Noise}

N250 amplitude was smaller for the APD group in quiet only compared with the TD control group. Both groups showed smaller N250 amplitudes in noise than in quiet. Unexpectedly, despite significant differences for N250 amplitude in quiet, N250 amplitude in noise was similar for the two groups. Table 1 shows that N250 approximately halved in amplitude in the TD control group with the introduction of noise and there was a smaller relative reduction in N250 amplitude in the children with APD. In a similar study comparing CAEPs in 7- to 13-year-old children with autism spectrum disorder versus a TD control group, Russo et al also saw more group differences in quiet than in noise. Russo et al found group differences for P1 latencies evoked by a synthetic speech stimulus $/ d a /$ in both quiet and in noise $(+5-$ dB signal-to-noise ratio), but P1-N250 amplitude only differed between groups in quiet. ${ }^{49}$

Overall, these results demonstrate that the current group of children with APD had poorer neurophysiological responses to the speech stimulus in quiet than the TD control children. Noise affected amplitudes differentially such that P1 was not significantly affected by the noise and N250 was reduced in noise by a lesser amount in the APD group than occurred for the TD control group. The addition of noise did not significantly affect the timing of the responses, which is consistent with some studies of children with language impairment. ${ }^{14,50} \mathrm{An}$ derson et al found that noise affected P1-N2 amplitudes of children ( 8 to 13 years) with learning problems whereas latencies were unaffected. ${ }^{50}$ Another study found that CAEP amplitudes were reduced in noise in TD children with no changes in latency, and a subset of children with learning problems similarly showed effects of noise on amplitudes only, consistent with the current study. ${ }^{13}$

\section{Are CAEPs Sensitive to Training Effects?}

\section{TEST-RETEST DIFFERENCES DURING THE PRETRAINING BASELINE FOR CHILDREN WITH APD}

$\mathrm{N} 1$ and P2 CAEP components increase in amplitude after active auditory discrimination training, ${ }^{32}$ although simple repeated exposure to a stimulus may be sufficient to induce plastic changes in $\mathrm{P} 2$ responses. ${ }^{51}$ To our knowledge there have not been any reports of changes to $\mathrm{CAEP}$ responses due to passive learning effects in children. In a previous study, ${ }^{4}$ active auditory training resulted in more robust CAEPs in noise. Hayes et al's study had a control group but no baseline measurements prior to the training phase. ${ }^{4}$ The current study showed that N250 amplitudes can change in the absence of any specific training and hence a control group alone is insufficient for establishing intervention effectiveness; establishing a stable pretraining baseline for electrophysiological (and other) outcome measurements is also important.

There are two alternative possibilities for the observed CAEP test-retest changes; one may be that exposure to the stimulus during testing over the two visits was enough to modify the amplitude of N250 through passive learning effects. Passive training effects such as this have 
been previously reported for $\mathrm{P} 2$ in adults, with increased P2 amplitudes observed after repeated CAEP testing. ${ }^{51,52}$ It is curious that instead of an increase in amplitude there was a decrease in amplitude for N250 in the current research. N250 amplitudes decreased with repeated testing and after the intervention phase in some groups in the current study. It is possible that the repeated exposure to the stimulus (in quiet and in noise) over the two baseline test sessions led to an emergence of $\mathrm{P} 2$. With the appearance of $\mathrm{P} 2$ in the maturing CAEP waveform, the late negativity in the waveform (N250) becomes correspondingly smaller. ${ }^{37}$ Because of the small amplitude and poor reliability of P2 it was not marked on the CAEP waveforms; however, the emergence of P2 could have influenced the amplitude of N250. This possibility is evident in the grand average CAEP waveforms (see Figs. 1 and 3).

Another possible explanation for test-retest changes in N250 amplitude may be habituation, which is defined as learning whereby stimulusspecific neuronal responses are reduced with a repeated stimulation. ${ }^{53,54}$ Previous research using functional magnetic resonance imaging has shown that repeated stimulation using musical stimuli for 43 minutes can reduce the magnitude of auditory cortical responses. ${ }^{53}$ This could account for N250 amplitude decreasing rather than showing no test-retest differences, as has been reported previously, ${ }^{1}$ or increasing in amplitude. $^{51}$

Differences in test populations, stimulus duration, and interstimulus interval between the current study and previous studies could have contributed to differences in test-retest effects across studies. Tremblay et al used longer-duration speech stimuli (484 to 756 milliseconds), a longer interstimulus interval (1910 milliseconds), and tested adults instead of children. ${ }^{1}$ Sheehan et al also tested adults and used longer stimulus durations (335 milliseconds); however, interstimulus interval (average onset asynchrony 1070 milliseconds) was similar to the current study. One difference is that Sheehan et al included sessions with active auditory training for some of the speech contrasts between CAEP recording sessions. ${ }^{51}$ In the current study, children were trained using various stimuli (tones, words, sentences) and tasks that were different from the CAEP test stimulus (speech phoneme in noise). Mutschler et al noted that repeated auditory stimulation in young adults can produce long-term decreases in brain activity (habituation), increased activity (sensitization), and sustained responses (no change). ${ }^{53}$

It is difficult to deduce specific factors underlying the significant reduction in N250 amplitudes during the baseline period. It would be useful in future studies to test for another baseline or other baselines to assess if CAEP amplitudes would decrease further or whether they would stabilize after three test sessions. Another idea would be to use intertwined stimuli (several different stimuli presented in pseudorandom order to avoid habituation effects). ${ }^{55}$ Regardless of the underlying mechanisms for the change in N250 amplitude, the findings are important for intervention research as the baseline changes in N250 amplitudes prior to any active training make it more difficult to interpret training effects.

\section{WAS A TRAINING EFFECT EVIDENT IN CAEP RESULTS FOR THE INTERVENTION GROUPS?}

Different types of training during the 6-week training phase of the study were associated with different amounts of change in N250 amplitude. Only the discrimination training group showed a statistically significant difference for N250 amplitude between visit 2 and 3, although there were some statistical trends $(p=0.07)$ for the language and the discrimination plus FM training groups. These results are not compelling and are limited by the relatively small sample sizes for the different types of training; however, they do suggest a possible greater impact of discrimination (bottom-up) training (compared to top-down language training) on CAEP amplitudes.

Discrimination training involved phonological awareness as well as frequency, intensity, and temporal resolution training. ${ }^{24}$ In a previous study, ${ }^{4}$ training using similar stimuli led to changes to CAEPs in noise. Hayes et al showed benefits of training in children with poor temporal processing (based on brainstem evoked response measurements). They found changes in CAEPs after training (N2 amplitude, consistent with the current study), but not 
brainstem responses. ${ }^{4}$ If they had performed two pretraining measurements, as we did, they also may have seen test-retest baseline differences, which would complicate the interpretation of their results.

There were no correlations between changes in CAEP results after the training period and improvements in the performance of the children with APD on the behavioral auditory processing tasks. ${ }^{24}$ Hence further research is needed to establish the functional significance of the observed passive and active auditory training effects on CAEP morphology in children with APD.

\section{SUMMARY AND CONCLUSIONS}

Noise affected CAEP amplitudes and latencies in children with and without APD. There were differences in CAEPs between TD children and children with APD, particularly for recordings in quiet. N250 amplitude changed during the short period of baseline testing, highlighting the importance of baseline testing prior to any intervention, in addition to having a no training and/or TD control group. Because CAEP recording parameters such as stimulus type and interstimulus interval and recording filter settings affect response amplitudes it is important that test protocols are standardized so that comprehensive norms can be established to determine whether CAEP amplitudes are significantly reduced and hence indicative of APD. Larger sample sizes are required to reliably establish effects of different types of training on CAEPs and related behavioral measures. The results did show that CAEPs are sensitive to passive auditory training effects and that it may be possible to produce reliable changes in N250 amplitude with certain active training approaches. There appeared to be a greater impact of discrimination based training on CAEPs, consistent with earlier studies such as Hayes et al, ${ }^{4}$ Menning et al, ${ }^{32}$ and Brattico et al, ${ }^{17}$ which also reported changes in CAEPs with discrimination training.

\section{REFERENCES}

1. Tremblay KL, Friesen L, Martin BA, Wright R. Test-retest reliability of cortical evoked potentials using naturally produced speech sounds. Ear Hear 2003;24(3):225-232

2. Cone B, Whitaker R. Dynamics of infant cortical auditory evoked potentials (CAEPs) for tone and speech tokens. Int J Pediatr Otorhinolaryngol 2013;77(7):1162-1173

3. Gilley PM, Sharma A, Dorman M, Martin K. Abnormalities in central auditory maturation in children with language-based learning problems. Clin Neurophysiol 2006;117(9):1949-1956

4. Hayes EA, Warrier CM, Nicol TG, Zecker SG, Kraus N. Neural plasticity following auditory training in children with learning problems. Clin Neurophysiol 2003;114(4):673-684

5. Cunningham J, Nicol T, Zecker S, Kraus N. Speech-evoked neurophysiologic responses in children with learning problems: development and behavioral correlates of perception. Ear Hear 2000;21(6):554-568

6. Gilley PM, Sharma A, Dorman M, Martin K. Developmental changes in refractoriness of the cortical auditory evoked potential. Clin Neurophysiol 2005;116(3):648-657

7. Sharma M, Purdy SC, Newall P, Wheldall K, Beaman R. Refractory effects on auditory-evoked responses in children with reading disorders. Neuroreport 2007;18(2):133-136

8. Hyde M. The N1 response and its applications. Audiol Neurootol 1997;2(5):281-307

9. Cone-Wesson B, Wunderlich J. Auditory evoked potentials from the cortex: audiology applications. Curr Opin Otolaryngol Head Neck Surg 2003; 11(5):372-377

10. Knight RT, Hillyard SA, Woods DL, Neville HJ. The effects of frontal and temporal-parietal lesions on the auditory evoked potential in man. Electroencephalogr Clin Neurophysiol 1980;50(1-2): 112-124

11. Bishop DVM, McArthur GM. Immature cortical responses to auditory stimuli in specific language impairment: evidence from ERPs to rapid tone sequences. Dev Sci 2004;7(4):F11-F18

12. Purdy SC, Kelly AS, Davies MG. Auditory brainstem response, middle latency response, and late cortical evoked potentials in children with learning disabilities. J Am Acad Audiol 2002;13(7): 367-382

13. Warrier CM, Johnson KL, Hayes EA, Nicol T, Kraus N. Learning impaired children exhibit timing deficits and training-related improvements in auditory cortical responses to speech in noise. Exp Brain Res 2004;157(4):431-441

14. Cunningham J, Nicol T, Zecker SG, Bradlow A, Kraus N. Neurobiologic responses to speech in noise in children with learning problems: deficits and strategies for improvement. Clin Neurophysiol 2001;112(5):758-767 
15. Breedin SD, Martin RC, Jerger S. Distinguishing auditory and speech-specific perceptual deficits. Ear Hear 1989;10(5):311-317

16. Jerger J, Musiek F. Report of the consensus conference on the diagnosis of auditory processing. J Am Acad Audiol 2000;11(9):467-474

17. Brattico E, Tervaniemi M, Picton TW. Effects of brief discrimination-training on the auditory N1 wave. Neuroreport 2003;14(18):2489-2492

18. Shahin A, Roberts LE, Pantev C, Trainor LJ, Ross B. Modulation of $\mathrm{P} 2$ auditory-evoked responses by the spectral complexity of musical sounds. Neuroreport 2005;16(16):1781-1785

19. Tremblay K, Kraus N, McGee T, Ponton C, Otis B. Central auditory plasticity: changes in the N1P2 complex after speech-sound training. Ear Hear 2001;22(2):79-90

20. Reinke KS, He Y, Wang C, Alain C. Perceptual learning modulates sensory evoked response during vowel segregation. Brain Res Cogn Brain Res 2003; 17(3):781-791

21. Friederichs E, Friederichs P. Clinical and auditory findings after one year application of a personal earlevel FM-device in children with symptoms of an auditory processing disorder: challenge of a possible new perspective. Journal of Educational Audiology 2005;12:31-36

22. Bishop DV. Research Review: Emanuel Miller Memorial Lecture 2012-neuroscientific studies of intervention for language impairment in children: interpretive and methodological problems. J Child Psychol Psychiatry 2013;54(3):247-259

23. Sharma M, Purdy SC, Kelly AS. Comorbidity of auditory processing, language, and reading disorders. J Speech Lang Hear Res 2009;52(3):706-722

24. Sharma M, Purdy SC, Kelly AS. A randomized control trial of interventions in school-aged children with auditory processing disorders. Int J Audiol 2012;51(7):506-518

25. Paavilainen $\mathrm{P}$, Alho K, Reinikainen K, Sams M, Näätänen R. Right hemisphere dominance of different mismatch negativities. Electroencephalogr Clin Neurophysiol 1991;78(6):466-479

26. Whiting KA, Martin BA, Stapells DR. The effects of broadband noise masking on cortical eventrelated potentials to speech sounds $/ \mathrm{ba} /$ and $/ \mathrm{da} /$. Ear Hear 1998;19(3):218-231

27. Billings CJ, Tremblay KL, Stecker GC, Tolin WM. Human evoked cortical activity to signalto-noise ratio and absolute signal level. Hear Res 2009;254(1-2):15-24

28. Kaplan-Neeman R, Kishon-Rabin L, Henkin Y, Muchnik C. Identification of syllables in noise: electrophysiological and behavioral correlates. J Acoust Soc Am 2006;120(2):926-933

29. Martin BA, Kurtzberg D, Stapells DR. The effects of decreased audibility produced by high-pass noise masking on $\mathrm{N} 1$ and the mismatch negativity to speech sounds /ba/and/da. J Speech Lang Hear Res 1999;42(2):271-286

30. Hillyard SA, Hink RF, Schwent VL, Picton TW. Electrical signs of selective attention in the human brain. Science 1973;182(4108):177-180

31. Picton TW, Hillyard SA. Human auditory evoked potentials. II. Effects of attention. Electroencephalogr Clin Neurophysiol 1974;36(2):191-199

32. Menning H, Roberts LE, Pantev C. Plastic changes in the auditory cortex induced by intensive frequency discrimination training. Neuroreport 2000;11(4):817-822

33. Shahin A, Roberts LE, Trainor LJ. Enhancement of auditory cortical development by musical experience in children. Neuroreport 2004;15(12): 1917-1921

34. Čeponienė R, Cummings A, Wulfeck B, Ballantyne A, Townsend J. Spectral vs. temporal auditory processing in specific language impairment: a developmental ERP study. Brain Lang 2009;110(3): 107-120

35. Sharma A, Dorman MF, Spahr AJ. A sensitive period for the development of the central auditory system in children with cochlear implants: implications for age of implantation. Ear Hear 2002; 23(6):532-539

36. Sussman E, Steinschneider M, Gumenyuk V, Grushko J, Lawson K. The maturation of human evoked brain potentials to sounds presented at different stimulus rates. Hear Res 2008;236(1-2): 61-79

37. Ponton C, Eggermont JJ, Khosla D, Kwong B, Don M. Maturation of human central auditory system activity: separating auditory evoked potentials by dipole source modeling. Clin Neurophysiol 2002;113(3):407-420

38. Lepistö T, Kujala T, Vanhala R, Alku P, Huotilainen $M$, Näätänen $R$. The discrimination of and orienting to speech and non-speech sounds in children with autism. Brain Res 2005;1066(1-2): 147-157

39. Lovio R, Näätänen R, Kujala T. Abnormal pattern of cortical speech feature discrimination in 6-yearold children at risk for dyslexia. Brain Res 2010; 1335:53-62

40. Jansson-Verkasalo E, Ceponiene R, Kielinen M, et al. Deficient auditory processing in children with Asperger syndrome, as indexed by eventrelated potentials. Neurosci Lett 2003;338(3): 197-200

41. Siegal M, Blades M. Language and auditory processing in autism. Trends Cogn Sci 2003;7(9): 378-380

42. Ouimet T, Foster NE, Tryfon A, Hyde KL. Auditory-musical processing in autism spectrum disorders: a review of behavioral and brain imaging studies. Ann N Y Acad Sci 2012;1252(1): 325-331 
43. O'Connor K. Auditory processing in autism spectrum disorder: a review. Neurosci Biobehav Rev 2012;36(2):836-854

44. Hyde KL, Samson F, Evans AC, Mottron L. Neuroanatomical differences in brain areas implicated in perceptual and other core features of autism revealed by cortical thickness analysis and voxel-based morphometry. Hum Brain Mapp 2010;31(4):556-566

45. Meyer M, Liem F, Hirsiger S, Jäncke L, Hänggi J. Cortical surface area and cortical thickness demonstrate differential structural asymmetry in auditoryrelated areas of the human cortex. Cereb Cortex May 3, 2013

46. Owen JP, Marco EJ, Desai S, et al. Abnormal white matter microstructure in children with sensory processing disorders. Neuroimage Clin 2013; 2:844-853

47. Liem F, Zaehle T, Burkhard A, Jäncke L, Meyer M. Cortical thickness of supratemporal plane predicts auditory N1 amplitude. Neuroreport 2012; 23(17):1026-1030

48. Schmithorst VJ, Holland SK, Plante E. Diffusion tensor imaging reveals white matter microstructure correlations with auditory processing ability. Ear Hear 2011;32(2):156-167

49. Russo N, Zecker S, Trommer B, Chen J, Kraus N. Effects of background noise on cortical encoding of speech in autism spectrum disorders. J Autism Dev Disord 2009;39(8):1185-1196

50. Anderson S, Chandrasekaran B, Yi HG, Kraus N. Cortical-evoked potentials reflect speech-in-noise perception in children. Eur J Neurosci 2010;32(8): 1407-1413

51. Sheehan KA, McArthur GM, Bishop DVM. Is discrimination training necessary to cause changes in the P2 auditory event-related brain potential to speech sounds? Brain Res Cogn Brain Res 2005; 25(2):547-553

52. Tremblay KL, Inoue K, McClannahan K, Ross B. Repeated stimulus exposure alters the way sound is encoded in the human brain. PLoS ONE 2010; 5(4):e10283

53. Mutschler I, Wieckhorst B, Speck O, et al. Time scales of auditory habituation in the amygdala and cerebral cortex. Cereb Cortex 2010;20(11): 2531-2539

54. Budd TW, Barry RJ, Gordon E, Rennie C, Michie PT. Decrement of the N1 auditory event-related potential with stimulus repetition: habituation vs. refractoriness. Int J Psychophysiol 1998;31(1): 51-68

55. Rankin CH, Abrams T, Barry RJ, et al. Habituation revisited: an updated and revised description of the behavioral characteristics of habituation. Neurobiol Learn Mem 2009;92(2):135-138 